Artículo científico

(Original paper)

\title{
COMPORTAMIENTO DEPREDADOR DE DOS ESPECIES DE ARAÑAS DEL GÉNERO PHONOTIMPUS (ARANEAE: PHRUROLITHIDAE)
}

\section{PREDATORY BEHAVIOR OF TWO SPIDER SPECIES OF THE GENUS PHONOTIMPUS (ARANEAE: PHRUROLITHIDAE)}

\author{
Gabriela GUADALUPe ANGULO ORDOÑES ${ }^{1}$, ARIANE DOR ${ }^{2}$, EMMANUEl Franco CAMPUZANO \\ GRANADOS ${ }^{1}$, GUILLERMO IBARRA NÚÑEZ ${ }^{1 *}$ \\ ${ }^{1}$ El Colegio de la Frontera Sur, Unidad Tapachula, Carretera Antiguo Aeropuerto km. 2.5, Tapachula, Chiapas, C. P. 30700 , \\ México. \\ ${ }^{2}$ Consejo Nacional de Ciencia y Tecnología comisionada a El Colegio de la Frontera Sur, Unidad Tapachula, Carretera Antiguo \\ Aeropuerto km. 2.5, Tapachula, Chiapas, C. P. 30700, México. \\ *Autor de correspondencia: <gibarra@ecosur.mx>
}

Recibido: 19/07/2018; aceptado: 22/01/2019; publicado en línea: 29/03/2019

Editor responsable: Arturo Bonet Ceballos

Angulo Ordoñes, G. G., Dor, A., Campuzano, G. E. F., Ibarra-Núñez, G. (2019) Comportamiento depredador de dos especies de arañas del género Phonotimpus (Araneae: Phrurolithidae). Acta Zoológica Mexicana (nueva serie), 35, 1-12. https://doi.org/10.21829/azm.2019.3502061

RESUMEN. Se estudió el comportamiento depredador de dos especies de arañas del género Phonotimpus (Araneae: Phrurolithidae) frente a cuatro tipos diferentes de presas potenciales (un Collembola, dos Hemiptera y un Psocoptera). Estas arañas habitan en la hojarasca del suelo de un bosque de niebla y de un cafetal en Chiapas, México. No existen estudios previos sobre el comportamiento de caza de esta familia de arañas. Debido al reducido tamaño de las arañas, el estudio se realizó en laboratorio, mediante videograbaciones de los encuentros con cada tipo de presa. A partir de las grabaciones se elaboró un catálogo de 16 actos de comportamiento que las arañas presentaron durante los encuentros con las presas, entre los que destacan el de "abanicar" y el de "ataque". Las grabaciones evidenciaron que ambas especies de arañas tuvieron una capacidad significativamente mayor de ataque y captura de los colémbolos que de los hemípteros y psocópteros, además de presentar un repertorio más variado de actos frente a los colémbolos y, para Phonotimpus pennimani un mayor nivel de actividad durante los encuentros con colémbolos. Así, nuestros resultados son similares a los de otros autores que estudiaron el consumo de presas en laboratorio, por dos especies distintas de Phrurolithidae, indicando que es altamente posible que en su hábitat natural los colémbolos sean parte importante de las presas de las Phrurolithidae. Las grabaciones también mostraron que ambas especies de arañas emplean dos estrategias de caza. Una es la emboscada, donde la araña permanece fija en un sitio por periodos relativamente prolongados, a la espera de la aproximación de una presa potencial, y la otra es la búsqueda activa, en la que la araña recorre activamente su entorno hasta que se topa con una presa potencial. De las dos estrategias, hubo un claro predominio de la emboscada, en cuanto su distribución de tiempos durante los encuentros.

Palabras clave: Caza; collembola; emboscada; búsqueda activa 
Angulo Ordoñes, G. G., Dor, A., Campuzano, G. E. F., Ibarra-Núñez, G. (2019) Predatory behavior of two spider species of the genus Phonotimpus (Araneae: Phrurolithidae). Acta Zoológica Mexicana (nueva serie), 35, 1-12. https://doi.org/10.21829/azm.2019.3502061

\begin{abstract}
We studied the predatory behavior of two spider species of the genus Phonotimpus (Araneae: Phrurolithidae) when confronted with four different types of potential prey (one Collembola, two Hemiptera and one Psocoptera). These spiders live in the soil leaf litter of a cloud forest and coffee orchards in Chiapas, Mexico. There are no previous studies of the hunting behavior for this spiders' family. Due to the small size of the spiders, the study was made in laboratory conditions with video recordings of the encounters with each prey type. From the analyses of the video recordings we established a catalog of 16 behavioral acts that the spiders displayed in the encounters with prey, among these we characterized "waving" and "attack". The recordings showed that both spider species had significant higher aptitudes to attack and capture springtails than hemipterans and psocopterans, the spiders displayed a richer behavioral repertoire when confronted the springtails and, for Phonotimpus pennimani a higher activity level when confronting the springtails. Thus, our results are similar to other studies on prey consumption in laboratory conditions by two different species of Phrurolithidae, pointing as highly possible that, in its natural habitat, springtails are an important part of the prey spectrum of the Phrurolithidae. The recordings also showed that both spider species employ two hunting strategies. One is ambushing, where the spider remains fixed in one place for relatively long periods, waiting for the approach of a potential prey, and the other is active searching, where the spider actively wanders its milieu until it encounters a potential prey. In terms of the distribution of times during encounters, ambushing predominated over active searching.
\end{abstract}

Key words: Hunting; collembola; ambushing; active searching

\title{
INTRODUCCIÓN
}

Las arañas son artrópodos depredadores muy abundantes en diferentes hábitats terrestres que capturan diferentes organismos, pero principalmente insectos, por lo que pueden ejercer una influencia importante en los ecosistemas de los que forman parte (Coddington \& Levi, 1991; Foelix, 2011). Las arañas han desarrollado diversas estrategias de depredación, que han servido como base para definir gremios (grupos de especies que aprovechan un mismo tipo de recurso de forma similar) los cuales pueden convivir en un mismo hábitat sin competir entre gremios (Uetz et al., 1999; Cardoso et al., 2011). Se ha estudiado el comportamiento depredador de diferentes especies, con énfasis en las que construyen redes, las que acechan mediante la vista a sus presas, y algunas de las que deambulan sobre la vegetación o sobre el suelo (Robinson et al., 1969; Jackson \& Pollard, 1996; Barth, 2001; Diaz-Fleischer, 2005; García, 2012). Sin embargo, hasta ahora se conoce poco sobre el comportamiento depredador de las especies que viven entre la hojarasca del suelo (García et al., 2018), a pesar de que se ha evidenciado el impacto de algunas de estas especies sobre la dinámica del reciclaje de la materia orgánica (Wise, 2004; Liu et al., 2015).

En las faldas del Volcán Tacaná, en Chiapas, se descubrieron recientemente dos especies de arañas del género Phonotimpus que habitan en la hojarasca (Chamé-Vázquez et al., 2018) y que pertenecen a la familia Phrurolithidae. Esta familia forma parte del clado de las Dionycha (Ramírez, 2014), el cual agrupa a varias familias de arañas que no utilizan redes para atrapar a sus presas, entre las que están Salticidae, Thomisidae y Lycosidae, cuyas estrategias de caza han sido ampliamente estudiadas, siendo unas acechadoras (localizan a distancia de la presa y se acercan sigilosamente), otras emboscadoras (permanecen estacionarias sobre una posición a la que se aproxima la presa) y otras corredoras de suelo (deambulan por el suelo hasta localizar una presa) (Uetz et al., 1999; Cardoso et al., 2011). Sin embargo, a la fecha no existen estudios de ningún tipo sobre el comportamiento de caza de las Phonotimpus en particular, ni de algún otro género de Phrurolithidae, aunque existe un estudio de laboratorio donde se 
analizó el consumo de presas (no el comportamiento mismo) en dos especies europeas de Phrurolithidae (Pekár \& Jarab, 2011).

Las dos especies de Phonotimpus estudiadas están entre las especies más comunes de la hojarasca de los sitios donde se colectaron (Chamé-Vázquez et al., 2018), por lo que resulta importante estudiar su comportamiento depredador para poder comprender su impacto potencial sobre otros habitantes de la hojarasca, y de manera indirecta su posible influencia en la dinámica del reciclaje de nutrientes del suelo. Así, este trabajo tiene por objetivos la caracterización del comportamiento depredador de dos especies de Phonotimpus (Phonotimpus talquian Chamé-Vázquez, Ibarra-Núñez y Jiménez, 2018 y Phonotimpus pennimani Chamé-Vázquez, Ibarra-Núñez y Jiménez, 2018) y sus variaciones frente a cuatro diferentes tipos de presas, estudiados en condiciones de laboratorio debido al tamaño de estas especies (2.0 a 2.8 $\mathrm{mm})$.

\section{MATERIALES Y MÉTODOS}

Phonotimpus talquian y P. pennimani son dos especies de arañas relativamente abundantes en la hojarasca de los lugares donde fueron colectadas. Phonotimpus talquian se colectó en un bosque mesófilo de montaña en el Ejido Talquián, Municipio de Unión Juárez, Chiapas $\left(15^{\circ} 05^{\prime} 15.00^{\prime \prime} \mathrm{N}, 92^{\circ} 05^{\prime} 56.00^{\prime \prime} \mathrm{O}\right.$, $2,010 \mathrm{msnm})$ y $P$. pennimani se colectó en un cafetal del Ejido Alpujarras, Municipio de Cacahoatán, Chiapas (15 4' 20.76" N y 92 9' 57.00" O, entre los 922-1,069 msnm) (Chamé-Vázquez et al., 2018). Mediante la revisión de hojarasca (de enero a agosto de 2017) se colectaron ejemplares vivos de ambas especies de arañas y de los insectos que cohabitan en ese sustrato, para llevarse al laboratorio donde se mantuvieron en condiciones controladas de temperatura $\left(26 \pm 1.4^{\circ} \mathrm{C}\right)$, humedad $(57 \pm 4.3 \%)$ y con un régimen de alrededor de 12 horas luz y 12 horas de oscuridad. Las arañas (sub-adultos y adultos) se colocaron individualmente en recipientes de plástico con tapa $(9 \times 9 \times 7 \mathrm{~cm})$, previamente provistos con una base $(2 \mathrm{~cm})$ de yeso mezclado con carbón activado para mayor retención de agua (García-Gómez et al., 2014). Cada tres días, el recipiente fue rociado con agua para mantener húmeda la base.

Entre los insectos colectados, se logró criar en laboratorio una especie del orden Collembola (Collembola sp.), la cual sirvió como alimento (solo durante una o dos ocasiones) para mantener a las arañas, esto con objeto de que las arañas estuvieran en iguales condiciones de alimentación antes de su primer encuentro con las presas experimentales. Los demás insectos se mantuvieron en recipientes de plástico, con una base húmeda y hojarasca.

Para los encuentros con las presas, las arañas empleadas fueron hembras adultas o sub-adultas ya que, entre las arañas, los machos adultos tienden a concentrarse en la búsqueda de parejas más que en la búsqueda de presas. Los artrópodos empleados como presas potenciales fueron Collembola sp., Delphacidae sp. (Hemiptera), Lygaeidae sp. (Hemiptera) y Caeciliidae sp. (Psocoptera), siempre la misma morfoespecie en cada caso, y elegidos por ser de talla similar a las arañas y por encontrarse con frecuencia en la hojarasca de los sitios de colecta de las arañas. De cada tipo de presa y para cada especie de araña se realizaron un mínimo de 30 encuentros, algunas arañas fueron utilizadas más de una vez, pero no con el mismo tipo de presa. Previo a cada encuentro, a cada araña se le retiró el alimento por un periodo de cuatro días a partir de su última captura, para estandarizar su nivel de hambre y motivar su impulso de caza.

Como arena de encuentros se empleó un recipiente de plástico rectangular $(10 \times 7.5 \mathrm{~cm})$ tapado con acrílico transparente. Las paredes del recipiente se cubrieron con una capa de FLUON (PTFE), creando una superficie resbaladiza para evitar que la araña y la presa treparan por las paredes y escaparan. En el fondo de la arena se colocó un papel absorbente ligeramente humedecido con el fin de simular, en algún grado, la textura de la hojarasca del hábitat de las arañas. Después de cada encuentro, el recipiente 
fue remplazado por otro previamente limpiado (con agua, alcohol y luego secado al aire) y con un papel nuevo. Cada encuentro fue grabado (entre las17:00 y las 21:00 horas) en video (archivo .mp4) con una cámara Sony (SLT-A37) para registrar con detalle las secuencias de actos conductuales de la araña y de la presa, con la cámara colocada unos $35 \mathrm{~cm}$ por arriba de la arena y ésta iluminada con luz roja, ya que los artrópodos de la hojarasca viven en condiciones de poca luz y porque arañas e insectos normalmente tienen muy baja o nula sensibilidad a la luz en el espectro de los 645 a $700 \mathrm{~nm}$, que corresponde a la luz roja (Foelix, 2011).

Para cada encuentro, araña y presa potencial se seleccionaron aleatoriamente, respetando los días sin alimento para cada araña. En la arena se introducía primero la araña, dejándola 10 minutos para permitir que se habituara y reducir el posible estrés por haber sido manipulada. Después se introducía (por el lado contrario a la araña) la presa correspondiente, de forma sutil para no perturbar a la araña. A partir de ese momento se comenzó el registro de datos hasta la captura de la presa, o hasta un máximo de 12 minutos para los casos en que no hubo actividad durante este tiempo. Cada grabación fue analizada usando un programa editor de video (Wondershare Filmora), para observar los movimientos en cámara lenta o incluso cuadro por cuadro $(0.033 \mathrm{~s})$, de manera que se pudieran registrar el momento en que se producía cada comportamiento y la duración correspondiente de estos.

Al concluir las grabaciones, para cada especie de Phonotimpus se registró el número de capturas realizadas por tipo de presa. Mediante la observación detallada de las videograbaciones se elaboró un catálogo de comportamientos (con sus descripciones), como base para analizar las secuencias de actos desplegados por cada especie de Phonotimpus frente a los diferentes tipos de presas ofrecidos en los encuentros, distinguiendo dos tipos básicos de comportamientos: eventos (actos de corta duración) y estados (actos con duración relativamente prolongada, como actividades prolongadas o posturas del cuerpo) (Martin \& Bateson, 1993).

Para presentar gráficamente las secuencias de actos y su variabilidad se elaboraron diagramas de flujo para cada tipo de encuentro (cada especie de araña con cada especie de presa) mostrando los actos desplegados con su frecuencia (en porcentajes) y las transiciones entre pares de actos, con sus correspondientes frecuencias. Para determinar si las arañas presentaban diferencias en comportamiento frente a los diferentes tipos de presas, de cada encuentro se determinaron y analizaron las posibles diferencias de: a) número de ataques, b) número de presas capturadas (resultado de las capturas exitosas, donde la araña dominó a las presas y las consumió), c) número de actos comportamentales diferentes, d) número total de actos por secuencia, e) duración de tres actos (con duración relativamente prolongada) que preceden el ataque (inmovilidad, desplazamiento por la arena y limpieza de apéndices). Asimismo, para analizar las estrategias de captura frente a cada tipo de presa, se determinó el tiempo (como porcentaje de la duración total del encuentro) en los estados de desplazamiento (estrategia de búsqueda activa) y en los que las arañas se mantenían fijas en un punto de la arena de encuentros (estrategia de emboscada). De igual forma, se determinó la proporción de casos de ataque que fueron precedidos por desplazamiento de la araña por la arena (estrategia de búsqueda activa) o por la araña en estado inmóvil o en limpieza, (estrategia de emboscada) por al menos 30 segundos antes del ataque. Los datos no cumplían con los supuestos necesarios para aplicar pruebas paramétricas, por lo que se usaron solamente pruebas no paramétricas, que según el caso fueron Chi-cuadrada, Kruskal-Wallis, Mann-Whitney y Wilcoxon.

\section{RESULTADOS}

Catálogo de actos. En los encuentros se registraron un total de 16 actos comportamentales diferentes para ambas especies, siendo 11 eventos y 5 estados (Cuadro 1). 
Se analizaron un total de 250 encuentros (videograbaciones), de los cuales 125 correspondieron a $P$. pennimani y 125 a $P$. talquian. Para ambas especies de arañas se registraron un total de 30 encuentros con Collembola sp., 34 con Delphacidae sp., 30 con Lygaeidae sp. y 31 con Caeciliidae sp.

Cuadro 1. Catálogo de los actos comportamentales de las especies de Phonotimpus observados durante los encuentros con los diferentes tipos de presas (+ eventos, \# estados).

\begin{tabular}{|c|c|c|}
\hline ACTO & CÓDIGO & DESCRIPCIÓN \\
\hline 1.-Inmovilidad & INM & \# Se mantiene en una posición sin mover sus apéndices. \\
\hline 2.-Desplazamiento & DES & \# Se desplaza por la arena de encuentro. \\
\hline 3.-Abanica & $\mathrm{ABA}$ & $\begin{array}{l}\text { + Mientras se mantiene en una posición, levanta una de sus patas } \\
\text { delanteras y la mueve de un lado a otro. }\end{array}$ \\
\hline 4.-Limpieza & LIM & $\begin{array}{l}\text { \# Mientras permanece en una posición, limpia sus apéndices, pasando uno } \\
\text { cada vez entre sus quelíceros. }\end{array}$ \\
\hline 5.-Aproximación & APR & $\begin{array}{l}\text { + Al desplazarse, pasa cerca de la presa, a dos cuerpos o menos de } \\
\text { distancia (con referencia al cuerpo de la araña). }\end{array}$ \\
\hline 6.-Detección & DET & $\begin{array}{l}\text { + Realiza un movimiento repentino (sobresalto: elevación ligera y breve } \\
\text { del cuerpo) cuando la presa se mueve cerca o tienen contacto directo. }\end{array}$ \\
\hline 7.-Orientación & OR & $\begin{array}{l}\text { + Gira su cuerpo (en dirección aproximada hacia la presa), después de } \\
\text { abanicar, o en respuesta a un movimiento de la presa. }\end{array}$ \\
\hline 8.- Expectativa & EXP & + Después de detectar a la presa, la araña permanece inmóvil brevemente. \\
\hline 9.-Acercamiento & ACE & $\begin{array}{l}\text { + Desplazamiento o movimiento corto (a menos de un cuerpo de distancia) } \\
\text { para acercarse a la presa. }\end{array}$ \\
\hline 10.-Ataque & ATA & $\begin{array}{l}\text { + Extiende las patas delanteras, abraza la presa y la jala hacia sus } \\
\text { colmillos. }\end{array}$ \\
\hline 11.-Captura & CAP & $\begin{array}{l}\text { + Después del ataque, la araña extiende sus patas delanteras mientras } \\
\text { sujeta a la presa con los colmillos. }\end{array}$ \\
\hline 12.-Transporte & TRA & \# Se desplaza con la presa capturada sostenida en sus colmillos. \\
\hline 13.-Consumo & $\mathrm{CON}$ & \# Permanece inmóvil con la presa sujeta en los colmillos. \\
\hline 14.-Evadir & EVA & + La araña se aleja de la presa. \\
\hline 15.-Falla & FALLA & + La araña no logra capturar a la presa después del ataque. \\
\hline 16.-Soltar & SOL & + La araña libera a la presa capturada. \\
\hline
\end{tabular}

Variación del comportamiento en función de las presas. Como resultado de esos encuentros, ambas especies de arañas realizaron un mayor número de ataques a los Collembola sp., mientras que los Caeciliidae sp. fueron la presa menos atacada (Cuadro 2). Estas diferencias en la propensión a los ataques por tipo de presa, se concretaron en diferencias en capturas exitosas, $P$. pennimani capturó 19 Collembola sp., 5 Delphacidae sp., 3 Lygaeidae sp. y 1 Caeciliidae sp., mientras que $P$. talquian capturó 12 Collembola sp., 5 Delphacidae sp., 3 Lygaeidae sp. y 1 Caeciliidae sp., diferencias que fueron significativas estadísticamente (Cuadro 2).

La discriminación entre las presas se detectó también en el número de actos comportamentales diferentes para ambas especies de arañas, $P$. pennimani exhibió un mayor número de actos diferentes frente a Collembola sp. y menos frente a las otras tres presas, Mientras que P. talquian exhibió un mayor número de actos diferentes frente a Collembola sp. y menos frente a Caeciliidae sp., pero no hubo diferencias entre los dos hemípteros, ni de estos con las otras dos presas (Cuadro 2). Para el número total de actos por secuencia solo se detectaron diferencias significativas frente a los tipos de presas para $P$. pennimani, que presentó el mayor número de actos frente a Collembola sp y Delphacidae sp., y el menor frente a Caeciliidae sp., mientras que no hubo diferencias de Lygaeidae sp. frente a los otros tipos de presas. 
Con respecto a la duración de los actos INM (inmovilidad), DES (desplazamiento) y LIM (limpieza), solo se encontraron diferencias significativas para $P$. pennimani en la duración de INM, con menores duraciones de este acto frente a Collembola sp. y mayores duraciones frente a Lygaeidae sp. y Caeciliidae sp., mientras que la duración de este acto frente a Delphacidae sp. no difirió de los otros tipos de presas. Los diagramas de flujo de los actos y transiciones de los diferentes tipos de secuencias muestran patrones similares de las dos especies de arañas frente a cada tipo de presa. Frente a los Collembola sp. (Fig. 1) las frecuencias de INM fueron menores a 18.2\%, las de LIM menores a 4.3\%, los DES menores a $10.3 \%$, los ATA y las CAP mayores a $8.4 \%$. Frente a los hemípteros Delphacidae sp. y Lygaeidae sp. (Figs. 2-3) las frecuencias de INM estuvieron en el rango de $24.6 \%$ a $27.3 \%$, las de LIM en el rango de $7.5 \%$ a $10 \%$, los DES en el rango de $10.9 \%$ a $18.1 \%$, los ATA en el rango de $4.5 \%$ a $7.3 \%$ y las CAP en el rango de $2.7 \%$ a $4.5 \%$. Frente a los Caeciliidae sp. (Fig. 4) las frecuencias de INM fueron mayores a $38.1 \%$, las de LIM mayores a $13.4 \%$, los DES mayores a $17.7 \%$, los ATA menores a $2.8 \%$ y las CAP menores a $1.5 \%$. Los diagramas también muestran un mucho menor número de transiciones entre actos frente a los Caeciliidae sp. (18 transiciones para $P$. pennimani y 28 para $P$. talquian), en comparación con los otros tipos de presas donde los diagramas son más complejos por el número de transiciones (de 46 a 53 transiciones para $P$. pennimani y de 44 a 52 para $P$. talquian).

Cuadro 2. Tipos de presas, número de encuentros y análisis de datos realizados para detectar diferencias en el comportamiento en función del tipo de presa. $\mathrm{Col}=$ Collembola sp., Del $=$ Delphacidae sp., Lyg $=$ Lygaeidae $\mathrm{sp}$ y $\underline{\mathrm{Cae}}=$ Caeciliidae sp. Pp. $=$ P. pennimani y Pt. $=$ P. talquian. Letras diferentes indican diferencias significativas.

\begin{tabular}{|c|c|c|c|c|c|}
\hline Presa & Col & Del & Lyg & Cae & Estadística \\
\hline No. de encuentros & 30 & 34 & 30 & 31 & \\
\hline Pp. Total de ataques & 21 & 8 & 5 & 2 & $\begin{array}{l}X^{2}=23.33, \text { g. } 1 .=3 \\
P<0.0001\end{array}$ \\
\hline Pt. Total de ataques & 14 & 8 & 8 & 2 & $\begin{array}{l}X^{2}=9.0, \text { g. } 1 .=3 \\
P<0.05\end{array}$ \\
\hline $\begin{array}{l}\text { Pp. presas capturadas / } \\
\text { no capturadas }\end{array}$ & $19 / 11$ & $5 / 29$ & $3 / 27$ & $1 / 30$ & $\begin{array}{l}X^{2}=39.28, \text { g. } 1 .=3, \\
P<0.0001\end{array}$ \\
\hline $\begin{array}{l}\text { Pt. presas capturadas / } \\
\text { no capturadas }\end{array}$ & $12 / 18$ & $5 / 29$ & $3 / 27$ & $1 / 30$ & $\begin{array}{l}X^{2}=16.73, \text { g. } 1 .=3, \\
P<0.001\end{array}$ \\
\hline $\begin{array}{l}\text { Pp. Actos diferentes por } \\
\text { secuencia }\end{array}$ & $\mathrm{a}$ & $\mathrm{b}$ & b & $\mathrm{b}$ & $\begin{array}{l}\mathrm{K}-\mathrm{W} \mathrm{H}=25.7, \text { g. } 1 .=3, \\
\mathrm{P}<0.0001\end{array}$ \\
\hline $\begin{array}{l}\text { Pt. Actos diferentes por } \\
\text { secuencia }\end{array}$ & $\mathrm{a}$ & $a b$ & $a b$ & $\mathrm{~b}$ & $\begin{array}{l}\mathrm{K}-\mathrm{W} \mathrm{H}=9.5, \text { g. } 1 .=3 \\
\mathrm{P}<0.025\end{array}$ \\
\hline $\begin{array}{l}\text { Pp. Total de actos por } \\
\text { secuencia }\end{array}$ & $\mathrm{a}$ & $\mathrm{a}$ & $a b$ & $\mathrm{~b}$ & $\begin{array}{l}\mathrm{K}-\mathrm{W} \mathrm{H}=21.5, \text { g. } 1 .=3, \\
\mathrm{P}<0.0001\end{array}$ \\
\hline Pt. Total de actos por secuencia & & & & & $\begin{array}{l}\text { K-W H }=5.99, \text { g. } 1 .=3, \\
P=0.1(\mathrm{~N} \mathrm{~S})\end{array}$ \\
\hline $\begin{array}{l}\text { Pp. Duración de INM por } \\
\text { secuencia }\end{array}$ & $\mathrm{a}$ & $a b$ & b & $\mathrm{b}$ & $\begin{array}{l}\mathrm{K}-\mathrm{W} \mathrm{H}=14.2, \text { g. } 1 .=3, \\
\mathrm{P}<0.005\end{array}$ \\
\hline $\begin{array}{l}\text { Pt. Duración de INM por } \\
\text { secuencia }\end{array}$ & & & & & $\begin{array}{l}\mathrm{K}-\mathrm{W} \mathrm{H}=4.1, \mathrm{~g} .1 .=3 \\
\mathrm{P}=0.2(\mathrm{~N} \mathrm{~S})\end{array}$ \\
\hline
\end{tabular}

Estrategias de caza. Respecto a la duración proporcional para cada secuencia de las arañas recorriendo la arena de encuentros (DES) y permaneciendo fija en un punto de la arena (INM + LIM), para ambas especies de arañas hubo una diferencia frente a todas las presas, con la duración de INM + LIM siendo significativamente mayor que la duración de DES (Cuadro 3). En cuanto a los comportamientos que precedieron los ataques de las arañas, para $P$. pennimani el $70 \%$ o más de los casos de ataque se produjeron cuando la araña estaba estacionada en un sitio (INM o LIM), mientras que la proporción más alta de ataques precedidos por desplazamiento fue solo de 30\%; para P. talquian el $80 \%$ o más de los 
casos de ataque se produjeron cuando la araña estaba fija en un sitio, mientras que la proporción más alta de ataques precedidos por desplazamiento fue de $20 \%$ (Cuadro 3 ).
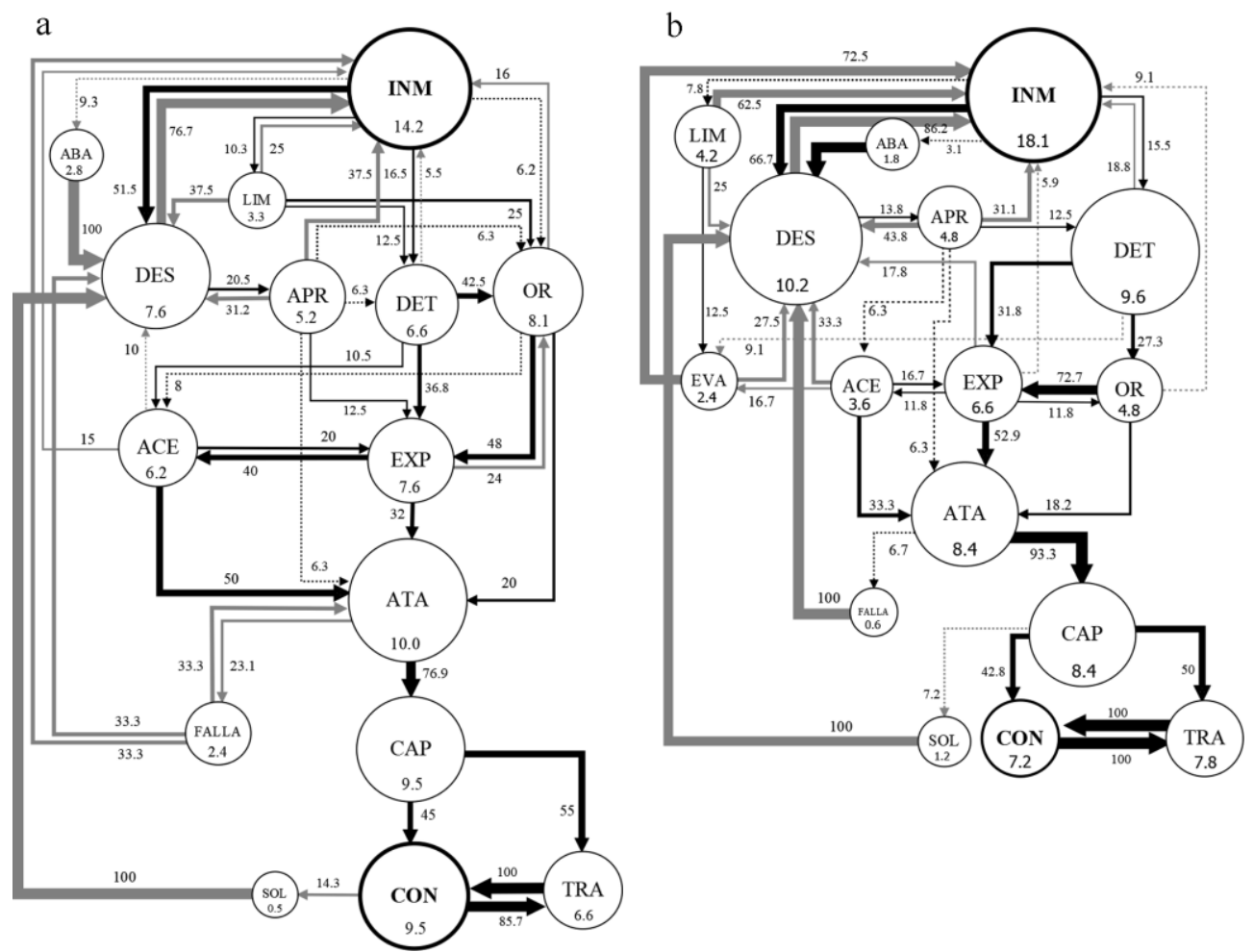

Figura 1. Diagramas de flujo de los actos de P. pennimani (a) y P. talquian (b) frente a Collembola sp. Círculos: su tamaño y número interior equivalen a la frecuencia (\%) de cada acto (abreviaturas del cuadro 1); círculos negros, actos donde concluyeron mayormente las secuencias. Flechas: su ancho equivale al porcentaje de transiciones entre dos actos; flechas negras, transiciones que concluyen en captura de la presa; flechas grises, transiciones que no concluyen en captura; flechas punteadas, transiciones menores a 10\%; las transiciones menores a $5 \%$ fueron omitidas.

Cuadro 3. Análisis del comportamiento previo al ataque de cada especie de araña (primera columna) frente a cada una de las presas ofrecidas (columnas 2 a 5): 3.1 y 3.2. Duración de tiempos de desplazamiento (DES) vs tiempo fijo sobre un sito en inmovilidad más limpieza, (INM + LIM). 3.3 y 3.4. Proporción de los encuentros donde se presentó INM o LIM antes del ataque (ATA) en comparación con los encuentros donde se presentó DES antes del ataque. Col $=$ Collembola sp., Del $=$ Delphacidae sp., Lyg $=$ Lygaeidae sp y Cae $=$ Caeciliidae sp. $\mathrm{Pp}=P$. pennimani y $\mathrm{Pt}=P$. talquian.

\begin{tabular}{lllll}
\hline & Col & Del & Lyg & Cae \\
\hline 3.1. Pp. Duración (mediana) & $418.9 / 7.5$ & $675.5 / 8.5$ & $696.5 / 14.5$ & $715.0 / 0.0$ \\
(INM + LIM) vs DES & $\mathrm{W}=459 ;$ & $\mathrm{W}=595 ;$ & $\mathrm{W}=463 ;$ & $\mathrm{W}=496 ;$ \\
& $\mathrm{z}=4.6589 ;$ & $\mathrm{z}=5.0896 ;$ & $\mathrm{z}=4.7429 ;$ & $\mathrm{z}=4.8636 ;$ \\
& $\mathrm{p}<0.001$ & $\mathrm{p}<0.001$ & $\mathrm{p}<0.001$ & $\mathrm{p}<0.001$ \\
& $563.1 / 4.1$ & $692.5 / 14.0$ & $708.5 / 0.0$ & $715.0 / 0.0$ \\
$\begin{array}{lll}\text { 3.2. Pt. Duración (mediana) } \\
\text { (INM + LIM) vs DES }\end{array}$ & $\mathrm{W}=452 ;$ & $\mathrm{W}=595 ;$ & $\mathrm{W}=465 ;$ & $\mathrm{W}=496 ;$ \\
& $\mathrm{z}=4.5150 ;$ & $\mathrm{z}=5.0866 ;$ & $\mathrm{z}=4.7867 ;$ & $\mathrm{z}=4.8639 ;$ \\
& $\mathrm{p}<0.001$ & $\mathrm{p}<0.001$ & $\mathrm{p}<0.001$ & $\mathrm{p}<0.001$ \\
$\begin{array}{l}\text { 3.3. Pp. \% de (INM + LIM) vs DES } \\
\text { como acto previo a ATA }\end{array}$ & $72.4 / 27.6$ & $87.5 / 12.5$ & $70 / 30$ & $100 / 0$ \\
$\begin{array}{l}\text { 3.4. Pt. \% de (INM + LIM) vs DES } \\
\text { como acto previo a ATA }\end{array}$ & $93.7 / 6.3$ & $80 / 20$ & $88.9 / 11.1$ & $100 / 0$ \\
\hline
\end{tabular}


a

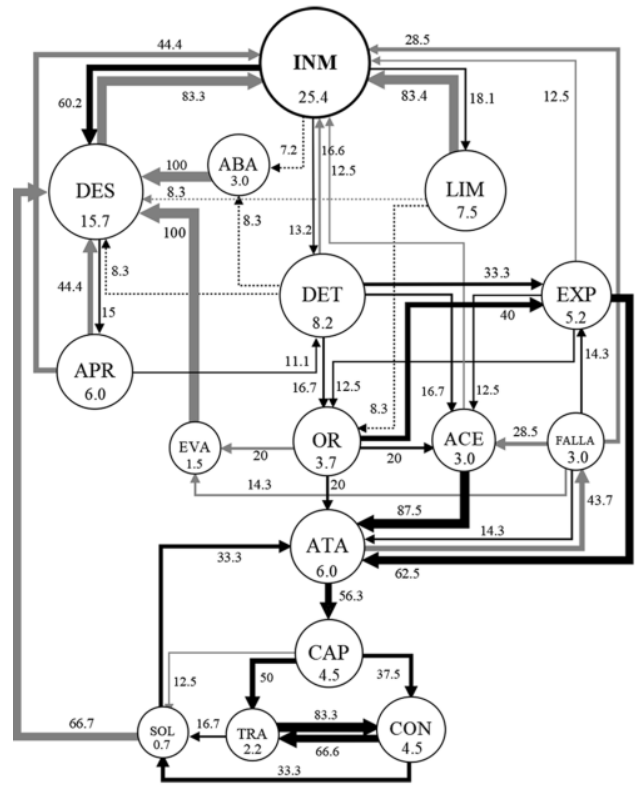

b

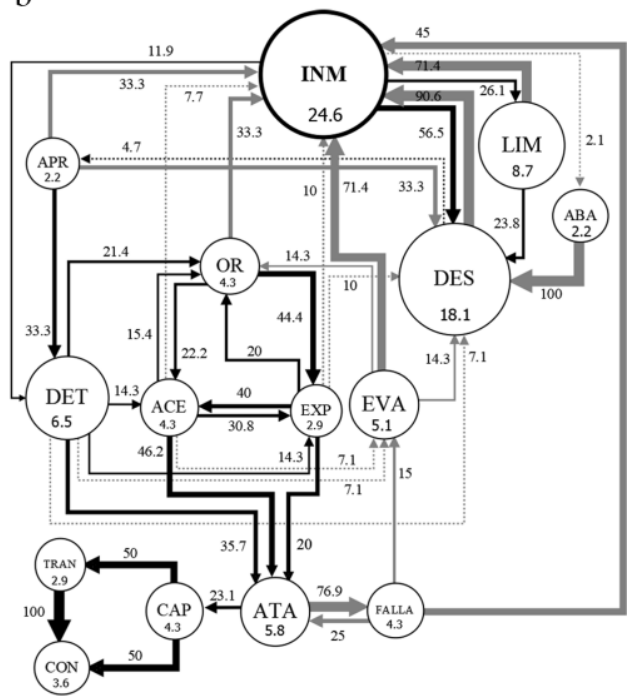

Figura 2. Diagramas de flujo de los actos de P. pennimani (a) y P. talquian (b) frente a Delphacidae sp. Círculos: su tamaño y número interior equivalen a la frecuencia (\%) de cada acto (abreviaturas del cuadro 1); círculos negros, actos donde concluyeron mayormente las secuencias. Flechas: su ancho equivale al porcentaje de transiciones entre dos actos; flechas negras, transiciones que concluyen en captura de la presa; flechas grises, transiciones que no concluyen en captura; flechas punteadas, transiciones menores a 10\%; las transiciones menores a $5 \%$ fueron omitidas.

\section{DISCUSIÓN}

Entre los comportamientos observados en las dos especies de Phonotimpus destaca uno que denominamos "abanicar", cuya función no es evidente. Este comportamiento es similar a uno que describe Forster (1982) para Trite planiceps (Salticidae) con los ojos cubiertos de pintura, donde al introducir la presa (díptero) al recipiente de la araña, esta eleva una o las dos patas anteriores y las mueve de lado a lado ("waving"), por lo que Forster infirió que ese comportamiento sirve a la detección de las vibraciones del aire que producen las moscas al batir sus alas. Barth (2001) ha demostrado que las arañas pueden detectar los movimientos del aire producidos por presas a través de las tricobótrias (setas sensibles a las vibraciones del aire) de sus patas. Así, es posible que el acto de "abanicar" le permita a las Phonotimpus utilizar sus patas anteriores para muestrear el aire en diferentes orientaciones, haciendo que sus tricobótrias puedan detectar vibraciones producidas por algún animal en movimiento en su entorno cercano, pero también es posible que al mover sus patas, las arañas estén tratando de percibir olores de presas potenciales, pues es sabido que las arañas son capaces de percibir olores a distancia, aunque se desconoce cuáles son los receptores que intervienen para esto (Ganske \& Uhl, 2018); esto requerirá de más estudios para confirmar esta hipótesis.

Otro comportamiento interesante es la forma en que se realiza el ataque, el cual se desencadena a corta distancia con la extensión de los dos pares de patas anteriores sobre la presa, seguida de su rápida contracción para acercar a esta a los quelíceros y la inmediata extensión una vez que la presa es asegurada. Comportamientos similares se han observado en otras arañas cazadoras errantes como Ctenidae y Lycosidae, las cuales poseen cojinetes de setas adhesivas en los tarsos que les sirven para sujetar y manipular a las presas en el ataque (Melchers, 1967; Rovner, 1980). Al igual que las dos familias 
mencionadas, las Phonotimpus poseen un cojinete de setas adhesivas en los tarsos (Chamé-Vázquez et al., 2018), que seguramente contribuyen para sujetar a las presas y atraerlas hacia los quelíceros.
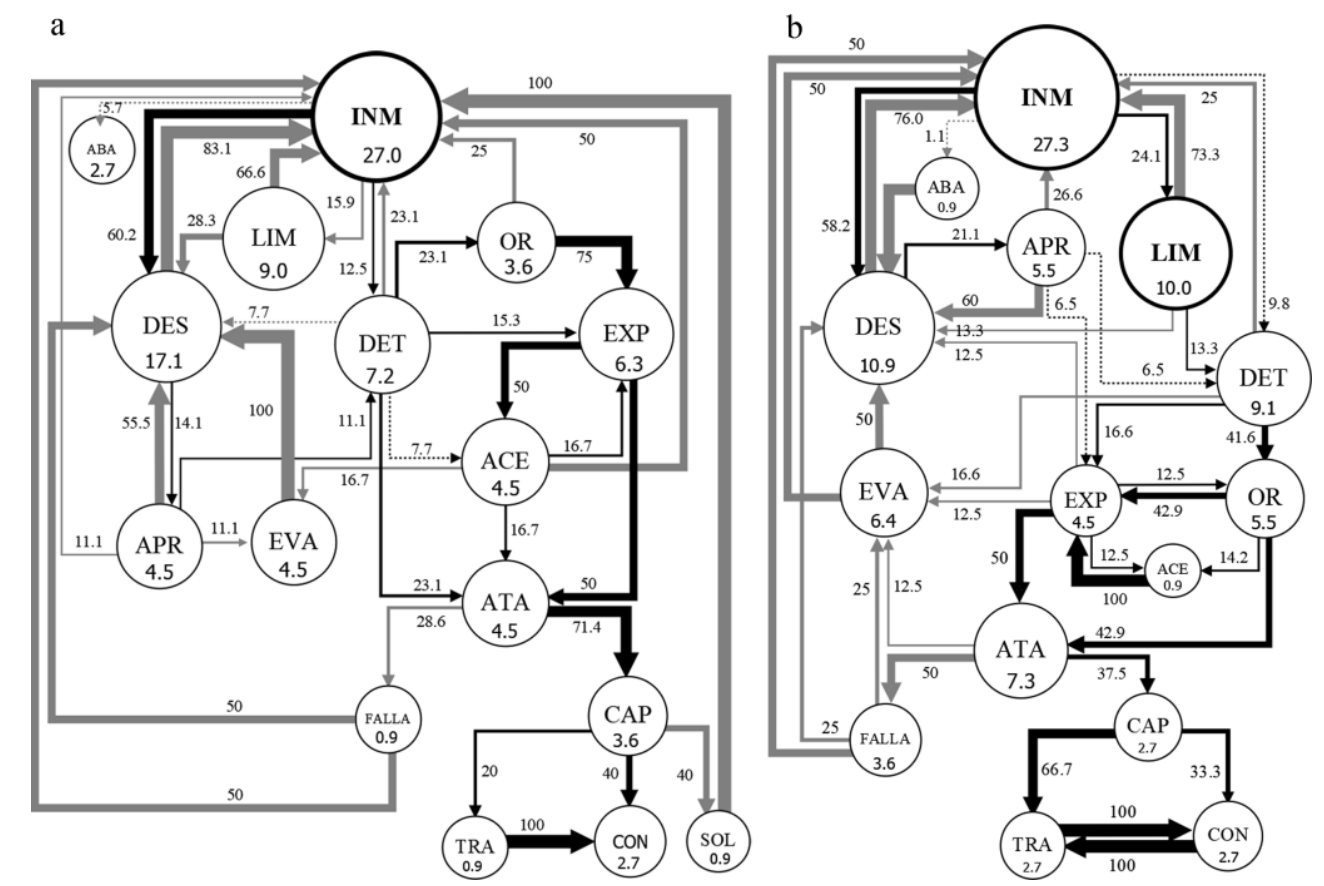

Figura 3. Diagramas de flujo de los actos de P. pennimani (a) y P. talquian (b) frente a Lygaeidae sp. Círculos: su tamaño y número interior equivalen a la frecuencia (\%) de cada acto (abreviaturas del cuadro 1); círculos negros, actos donde concluyeron mayormente las secuencias. Flechas: su ancho equivale al porcentaje de transiciones entre dos actos; flechas negras, transiciones que concluyen en captura de la presa; flechas grises, transiciones que no concluyen en captura; flechas punteadas, transiciones menores a 10\%; las transiciones menores a $5 \%$ fueron omitidas.

Las diferencias detectadas revelan que las dos especies de arañas son capaces de discriminar entre los tipos de presas ofrecidas, al mostrar diferencias en su comportamiento que se evidencian en los patrones mostrados en los diagramas de flujo de actos, así como en una mayor eficiencia para atacar y capturar a los Collembola sp. que a las otras presas, y un mayor despliegue de actos diferentes frente a los Collembola sp. que frente a las otras presas, revelando mayor variabilidad en su comportamiento con ese tipo de presa. Sin embargo, esa capacidad de discriminación no es la misma para las dos especies de arañas, ya que las $P$. pennimani presentaron una mayor actividad (un mayor número total de actos y una menor duración de la inmovilidad) frente a los Collembola sp. que, frente a las otras presas, pero no se detectó esto para $P$. talquian, la cual no mostró diferencias en el nivel de actividad (número total de actos y duración de la inmovilidad) entre todas las presas. Las otras presas (hemípteros y Caeciliidae sp.) solo fueron atacados y capturados en unos pocos casos por ambas especies de arañas, evidenciando una reacción baja hacia los hemípteros y casi nula frente a los Caeciliidae sp., indicando que, a pesar de ser comunes en el hábitat natural de ambas arañas, no parecen estar entre las presas habituales de estas especies. Se pudiera suponer que la mayor capacidad de captura de los Collembola sp. por ambas especies de arañas, se deriva de su experiencia previa con esta especie de presa, cuando se le alimentó en el periodo previo al inicio de las observaciones, sin embargo esta experiencia se limitó a una o dos ocasiones con este tipo de presa, lo cual no es definitivo para desarrollar su preferencia, ni mucho menos para desarrollar la eficiencia de captura que se observó en comparación con los otros tipos de presas. Es sabido que algunas arañas errantes (e. g. Lycosidae, Salticidae y Thomisidae) son capaces de capturar colémbolos, pero estos no están entre sus presas principales (Hallander, 1970; Guseinov et al., 2004; Huseynov et al., 2005; 
Guseinov, 2006). Sin embargo, en pruebas de consumo en laboratorio Triaeris stenaspis (Oonopidae), Liophrurillus flavitarsis (Phrurolithidae) y Phrurolithus festivus (Phrurolithidae) sí tuvieron a los colémbolos como presas principales, o casi exclusivas (Pekár \& Jarab, 2011; Korenko et al., 2014). Así, los resultados de este trabajo parecen indicar que las Phonotimpus comparten esta preferencia por los colémbolos, observada en otras Phrurolithidae, sin llegar a ser especialistas de estos como parece ser el caso de Triaeris stenaspis.

Respecto a las estrategias de caza, la comparación de las duraciones proporcionales empleadas por las arañas para desplazarse o mantenerse en un sitio durante los encuentros, indica claramente que la estrategia de caza más empleada fue la emboscada. Esto se ve confirmado con el dato de que la mayoría de los ataques a las presas se produjeron con la araña estacionada en un punto de la arena de encuentros. Sin embargo, las observaciones también confirman que las Phonotimpus pueden emplear la estrategia de búsqueda activa, que detectan una presa al ir recorriendo su entorno para aproximarse rápidamente y atacarlas enseguida. Un patrón similar de distribución de tiempo, entre estacionario y móvil, fue observado en Pardosa agrestis en campo (Samu et al., 2003), estas arañas pasaron la mayor parte del tiempo estacionadas ("waiting") en distintos sitios (90\%), alternando con lapsos cortos de desplazamiento a otros sitios donde permanecieron fijas la mayor parte del tiempo. Adicionalmente, en ese estudio se observó que los ataques a las presas solo se desencadenaron cuando las arañas estaban estacionadas y una presa se acercaba a pocos centímetros de ella.
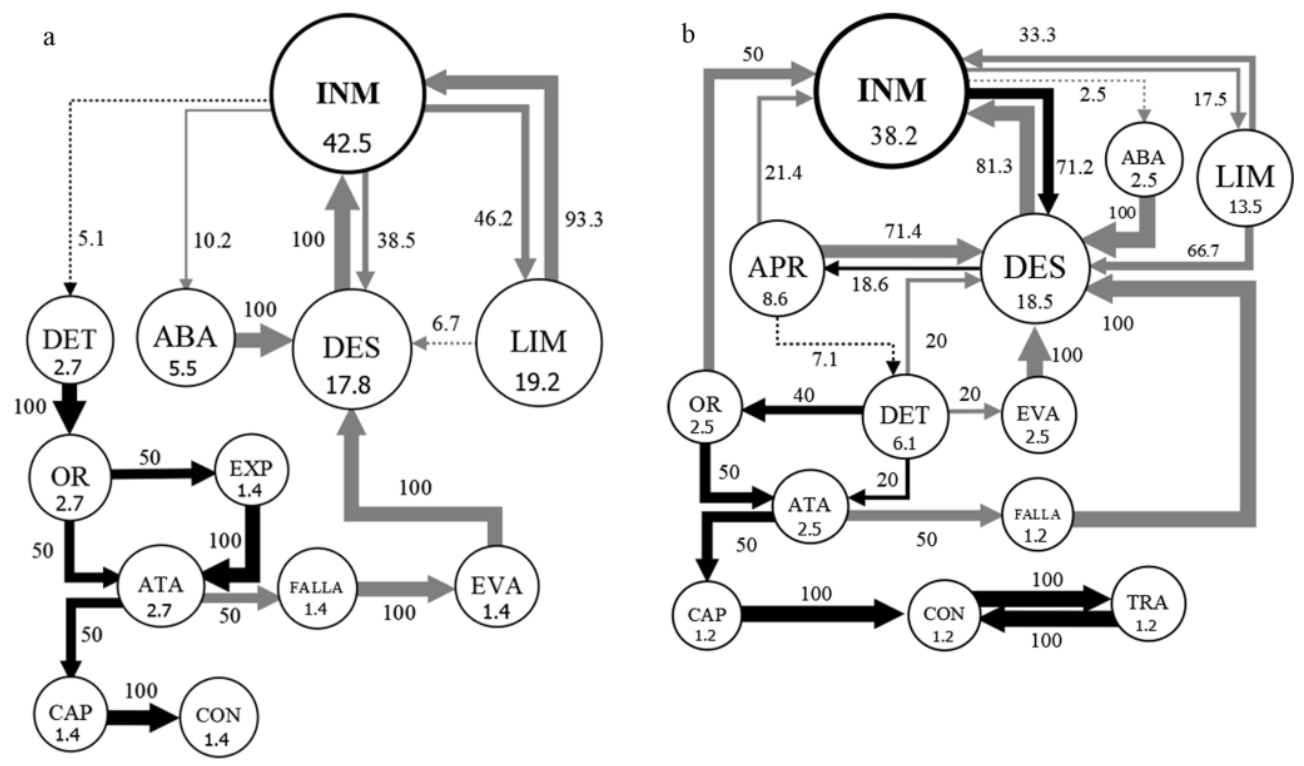

Figura 4. Diagramas de flujo de los actos de P. pennimani (a) y P. talquian (b) frente a Caeciliidae sp. Círculos: su tamaño y número interior equivalen a la frecuencia (\%) de cada acto (abreviaturas del cuadro 1); círculos negros, actos donde concluyeron mayormente las secuencias. Flechas: su ancho equivale al porcentaje de transiciones entre dos actos; flechas negras, transiciones que concluyen en captura de la presa; flechas grises, transiciones que no concluyen en captura; flechas punteadas, transiciones menores a 10\%; las transiciones menores a $5 \%$ fueron omitidas.

El predominio de la estrategia de emboscada en $P$. pennimani y $P$. talquian indica que estas arañas dependen en gran medida de la movilidad de sus presas por una parte y por otra, de la localización de sitios atractivos para estas, por lo cual deben mantener un patrón de desplazamientos cada cierto lapso de tiempo, para incrementar las probabilidades de encontrarse con una presa. 
En conclusión, podemos decir que $P$. pennimani y $P$. talquian son más eficientes para capturar Collembola sp., que las otras presas ofrecidas en este estudio; esa eficiencia pudiera estar relacionada con factores de defensa de las presas, pero también con posibles adaptaciones de las arañas para capturar a este tipo de presas. Sin embargo, las dos especies de Phonotimpus estudiadas fueron capaces de capturar a otras presas, aunque con mucha menor eficiencia. Asimismo, se observó que las dos especies de Phonotimpus son capaces de exhibir dos estrategias de depredación, emboscada y búsqueda activa, con un claro predominio de la primera.

Agradecimientos. A los revisores (Dr. V Dinesh Rao y un revisor anónimo) por sus sugerencias que contribuyeron a mejorar este trabajo. A Héctor Montaño Moreno y David Chamé Vázquez (ECOSUR) por su apoyo en las colectas de campo. A David Chamé Vázquez por sus consejos en el establecimiento y mantenimiento de la cría de colémbolos. A Fernando Dorantes Nieto por su apoyo en los análisis estadísticos. Al CONACYT por la beca otorgada a G.G.A.O. durante sus estudios de maestría.

\section{LITERATURA CITADA}

Barth, F. G. (2001) A spider's world: senses and behavior. Berlin, Heidelberg: Springer-Verlag. 394 pp.

Cardoso, P., Pekár, S., Jocqué, R., Coddington, J. A. (2011) Global patterns of guild composition and functional diversity of spiders. PLoS ONE. 6 (6), e21710, 1-10.

https://doi.org/10.1371/journal.pone.0021710

Chamé-Vázquez, D., Ibarra-Núñez, G., Jiménez, M. L. (2018) Redescription of Phonotimpus separatus Gertsch \& Davis, 1940 (Araneae: Phrurolithidae) and description of two new species of Phonotimpus from Mexico. Zootaxa, 4407 (2), 213-228.

https://doi.org/10.11646/zootaxa.4407.2.3

Coddington, J. A., Levi, H. W. (1991) Systematics and Evolution of Spiders (Araneae). Annual Review of Ecology and Systematics, 22, 565-592.

Díaz-Fleischer, F. (2005) Predatory behaviour and prey-capture decision-making by the web-weaving spider Micrathena sagittata. Canadian Journal of Zoology, 83 (2), 268-273. https://doi.org/10.1139/z04-176

Foelix, R. F. (2011) Biology of spiders (3rd ed.). New York, Oxford University Press. 419 pp.

Forster, L. M. (1982) Non-visual prey-capture in Trite planiceps, a jumping spider (Araneae, Salticidae). Journal of Arachnology, 10 (2), 179-183. https://doi.org/10.2307/3705341

Ganske, A. S., Uhl, G. (2018). The sensory equipment of a spider - A morphological survey of different types of sensillum in both sexes of Argiope bruennichi (Araneae, Araneidae). Arthropod Structure \& Development, 47 (2), 144-161. https://doi.org/10.1016/j.asd.2018.01.001

García, L. F. (2012) Comportamiento predador en dos arañas del género Oecobius (Araneae, Oecobiidae). Tesis de Maestría. Universidad de la República de Uruguay. 102 pp.

García, L. F., Viera, C, Pekár, S. (2018) Comparison of the capture efficiency, prey processing, and nutrient extraction in a generalist and a specialist spider predator. The Science of Nature, 105, 30. https://doi.org/10.1007/s00114-018-1555-z

García-Gómez, A., Callejas-Chavero, A., Castaño-Meneses G. (2014) Importancia de cultivos para el estudio de colémbolos (Hexapoda: Collembola) de hábitos edáficos. Dugesiana, 21 (2), 103-106.

Guseinov, E. F. (2006) The prey of a lithophilous crab spider Xysticus loeffleri (Araneae, Thomisidae). Journal of Arachnology, 34 (1), 37-45. https://doi.org/10.1636/M02-14.1 
Guseinov, E. F., Cerveira, A. M., Jackson, R. R. (2004) The predatory strategy, natural diet, and life cycle of Cyrba algerina, an araneophagic jumping spider (Salticidae: Spartaeinae) from Azerbaijan. New Zealand Journal of Zoology, 31 (4), 291-303. https://doi.org/10.1080/03014223.2004.9518382

Hallander, H. (1970) Prey, cannibalism and microhabitat selection in the wolf spiders Pardosa chelata O. F. Müller and P. pullata Clerck. Oikos, 21 (2), 337-340. http://www.jstor.org/stable/354

Huseynov, E. F., Cross, F. R., Jackson, R. R. (2005) Natural diet and prey-choice behaviour of Aelurillus muganicus (Araneae: Salticidae), a myrmecophagic jumping spider from Azerbaijan. Journal of Zoology, 267 (2), 159-165. https://doi.org/10.1017/S0952836905007363

Jackson, R. R., Pollard, S. D. (1996) Predatory behavior of jumping spiders. Annual Review of Entomology, 41 (1), 287-308. https://doi.org/10.1146/annurev.en.41.010196.001443

Korenko, S., Hamouzová, K., Pekár, S. (2014) Trophic niche and predatory behavior of the goblin spider Triaeris stenaspis (Oonopidae): a springtail specialist? Journal of Arachnology, 42 (1), 74-78. https://doi.org/10.1636/Hi12-90.1

Liu, S., Chen, J., Gan, W., Schaefer, D., Gan, J., Yang, X. (2015) Spider foraging strategy affects trophic cascades under natural and drought conditions. Scientific Reports, 5, 1-9. https://doi.org/10.1038/srep12396

Martin, P., Bateson, P. (1993) Measuring behaviour. An introductory guide (2nd ed.). Cambridge University Press. Cambridge, U. K. 222 pp.

Melchers, M. (1967) Der Beutefang von Cupiennius salei Keyserling (Ctenidae). Zeitschrift Für Morphologie Und Ökologie Der Tiere, 58 (3), 321-346. http://www.jstor.org/stable/43262

Pekár, S., Jarab, M. (2011) Life-history constraints in inaccurate Batesian myrmecomorphic spiders (Araneae: Corinnidae, Gnaphosidae). European Journal of Entomology, 108 (2), 255-260. https://doi.org/10.14411/eje.2011.034

Ramírez, M. J. (2014) The morphology and phylogeny of Dionychan spiders (Araneae: Araneomorphae). Bulletin of the American Museum of Natural History, 390, 1-374. https://doi.org/10.1206/821.1

Robinson, M. H., Mirick, H., Turner, O. (1969) The predatory behavior of some araneid spiders and the origin of immobilization wrapping. Psyche (New York), 76 (4), 487-501. https://doi.org/10.1155/1969/68029

Rovner, J. S. (1980) Morphological and ethological adaptations for prey capture in wolf spiders (Araneae, Lycosidae). Journal of Arachnology, 8 (3), 201-215. https://doi.org/10.2307/3705002

Samu, F., Szirányi, A., Kiss, B. (2003) Foraging in agricultural fields: local "sit-and-move" strategy scales up to risk-averse habitat use in a wolf spider. Animal Behaviour, 66 (5), 939-947. https://doi.org/10.1006/anbe.2003.2265

Uetz, G. W., Halaj, J., Cady, A. B. (1999) Guild structure of spiders in major crops. Journal of Arachnology, 27 (1), 270-280. https://doi.org/10.2307/3705998

Wise, D. H. (2004) Wandering spiders limit densities of a major microbi-detritivore in the forest-floor food web. Pedobiologia, 48 (2), 181-188. https://doi.org/10.1016/j.pedobi.2003.12.001 